Ingeniería Investigación y Tecnología. Vol.XI. Núm.2. 2010 233-245,

ISSN 2594-0732, FI-UNAM (artículo arbitrado)

DOI: http://dx.doi.org/10.22201/fi.25940732e.2010.11n2.019

\title{
Cuantificación de energía de una planta mareomotriz
}

\section{Tidal Power Plant Energy Estimation}

\author{
López-González J. \\ Instituto de Ingeniería \\ Universidad Nacional Autónoma de México. \\ E-mail:JlopezGo@iingen.unam.mx \\ Hiriart-Le Bert G. \\ Instituto de Ingeniería \\ Universidad Nacional Autónoma de México. \\ E-mail:gerardo367@yahoo.com.mx \\ Silva-Casarín R. \\ Instituto de Ingeniería \\ Universidad Nacional Autónoma de México. \\ E-mail:RSilvaC@iingen.unam.mx
}

(Recibido: agosto de 2008; reevaluado: agosto de 2009; aceptado: agosto de 2009)

\section{Resumen}

Se presenta una metodología que permite estimar de manera rápida y sencilla el potencial de energía que puede ser extraído a través de una planta mareomotriz. La evaluación se realiza utilizando un nomograma normalizado que es función del área del embalse versus la capacidad eléctrica instalada, para así obtener el potencial energético en una zona en particular. Los resultados contemplan dos formas de operación, una llamada en flujo y otra en flujo-reflujo, además, dos esquemas de embalse (sencillo y doble embalse). Para la elaboración del nomograma normalizado se utilizaron los resultados numéricos de la simulación de múltiples plantas mareomotrices bajo diferentes escenarios durante un año de operación. Los escenarios fueron establecidos variando la potencia instalada, condiciones de aprovechamiento en flujo, reflujo o ambos, así como embalse sencillo o doble con información del nivel del mar cada 15 min. Para validar el modelo se utilizó información de la mareomotriz de La Rance, Francia, que incluye datos sobre la producción, potencia instalada, propiedades de las turbinas y niveles de la marea, obteniéndose una muy buena correlación entre los resultados del modelo numérico y los reportados en varios reportes técnicos.

Descriptores: energía mareomotriz, energía renovable, potencia instalada.

\section{Abstract}

In this paper a methodology is presented which allows a quick and simple means of estimating the potential energy that can be obtained from a tidal power plant. The evaluation is made using a normalised nomograph, which is a function of the area of the tidal basin against the electricity installed capacity to thus obtain the potential energy for any 
DOI: http://dx.doi.org/10.22201/fi.25940732e.2010.11n2.019

Cuantificación de energía de una planta mareomotriz

location. The results describe two means of operation, one of "flow tide" and the other "flow-ebb tides", with two tidal basin systems operating: single and double reservoir systems. To obtain the normalised nomograph the numerical results for simulations of several tidal power plants under differing operational conditions over a period of one year. These conditions were established by varying the electricity installed capacity, the hydraulic conditions in "flow tide", "ebb tides" or both and with single or double reservoir systems and using sea level information taken every 15 minutes. To validate the model information from the tidal power plant at Rance, France, was used, which includes data concerning production, electricity installed capacity, turbine characteristics and tidal ranges. A very good correlation was found between the results of the numerical model and those reported in various technical reports.

Keywords: Tidal power, renewable energy, installed capacity.

\section{Introducción}

La evolución de las sociedades a nivel mundial ha estado acompañada de una dependencia de dispositivos eléctricos que demandan grandes cantidades de energía para trabajar. En los últimos años, el crecimiento de la población de las grandes ciudades, particularmente las industrializadas, es del orden del $2 \%$ más alto que el promedio mundial y el incremento de demanda de energía reporta la misma tendencia. A medida que las sociedades incrementan su confort en el estilo de vida, la demanda de energía es mayor, lo cual se refleja en el consumo energético relativo de los países más industrializados, que con una población del $25 \%$ consume el $75 \%$ de la energía mundial (Fells, 1990).

Una forma de producir energía eléctrica es usando combustibles fósiles, pero el impacto negativo que ocasiona (calentamiento global) y los altos costos del mismo (Ballenilla, 2004), han hecho que muchos gobiernos de diferentes países realicen esfuerzos muy importantes en la utilización de fuentes renovables de energía (Dincer, 2000). Sin duda, el océano es una de estas fuentes de energía renovable, ya que en él, se concentra la energía del sol, viento y las fuerzas de atracción del sol y la luna. Se puede decir que casi todas las fuentes de energía provienen del sol; la energía solar proporciona un flujo continuo de energía necesaria para el crecimiento de cosechas vía fotosíntesis, gradiente térmico en la superficie terrestre y en el mar, el cual deriva en vientos, que a su vez generan oleaje y por supuesto lluvia, factor primordial para la generación hidroeléctrica, la cual es una de las primeras formas de energía renovable.

Los científicos han realizado numerosos estudios para conocer y aprovechar la energía oceánica; sin embargo, en algunos casos los fondos para realizar tales investigaciones no son suficientes para completar adecuadamente los estudios y definir la mejor manera de aprovechar el potencial de esta fuente de energía (Fells, 1990). El entendimiento científico de los fenómenos físicos y las formas de energía es la primera parte de la explotación de la energía. La construcción de centrales eléctricas en el océano, es un poco más difícil de llevar a cabo, debido a la variabilidad de los recursos y el alto costo de construcción y mantenimiento.

La idea de aprovechar la energía contenida en las mareas no es nueva. Desde 1890, varias ideas y patentes surgieron y la mayoría de ellas eran francesas. La idea de usar la energía del océano para producir electricidad es más antigua; a pesar de que Romanoski en 1950, analizó la energía térmica del oleaje y de mareas, la energía mareomotriz ha sido usada por cientos de años antes (Charlier et al., 1993) y (Charlier, 1982).

El océano tiene una cantidad enorme de energía, muchos estudios muestran que esta cantidad es de alrededor de 5 millones de MW, pero por supuesto la realidad es que solo una fracción mínima de esta energía es posible aprovechar.

\section{Modos de operación}

Una instalación mareomotriz con un embalse puede operar cuando:

1) La marea está entrando (flujo),

2) Cuando la marea está saliendo (reflujo) y

3) En los dos sentidos (Flujo y reflujo), adicionalmente se le puede instalar un sistema de bombeo de agua en horas no pico para aumentar el desnivel (Charlier et al., 1993).

1. Modo de Flujo: Consiste en la generación de energía eléctrica cuando la marea está ascendiendo y el nivel de agua en el embalse es inferior al nivel de la marea; entonces, la generación de energía se 
DOI: http://dx.doi.org/10.22201/fi.25940732e.2010.11n2.019

López-González J., Hiriart-Le Bert G. y Silva-Casarín R.

realiza cuando el flujo del agua es del mar hacia el embalse.

2. Modo de Reflujo. Consiste en la generación de energía eléctrica cuando la marea está descendiendo, de modo que cuando la marea está ascendiendo, se permite el paso del agua hacia el embalse por medio de unas compuertas, hasta alcanzar el nivel máximo, para permitir luego el paso del agua por las turbinas cuando la marea está descendiendo y el flujo del agua es del embalse hacia el mar. Generalmente, el paso del agua por las turbinas no ocurre hasta que se tiene cierta diferencia entre el nivel del embalse y el nivel de marea fuera del mismo.

3. Modo de flujo y reflujo. Este tipo de generación de energía consiste en aprovechar el ascenso y descenso de la marea, mediante una combinación de los dos modos anteriores.

Con estos modos de operación hay intervalos en los que no se tiene generación, debido a que los niveles del agua dentro y fuera del embalse son los mismos, para lo cual existe el esquema de doble embalse, en el cual, en uno de los embalses sólo se permite la entrada de agua, cuando el nivel en el mismo es menor que la marea (embalse alto) y en el otro sólo se permite la salida del agua cuando su nivel de agua es mayor que la marea y los generadores se encuentran instalados entre estos dos embalses en los cuales, dependiendo del volumen del embalse y la potencia instalada siempre tendrá un desnivel para generar la mayor parte del tiempo.

\section{Sitios}

La cantidad de energía que es posible aprovechar, está directamente relacionada con la amplitud de la marea, pero también es importante la cantidad de turbinas y el área del embalse. La correcta combinación de estos tres factores dará como resultado mayores beneficios a través de una mayor eficiencia.

Hay varios lugares en el mundo que presentan las condiciones óptimas para aprovechar las mareas para la generación de energía, con variaciones de hasta $16 \mathrm{~m}$. Hoy en día, el desarrollo de las turbinas sólo permite aprovechar carreras de marea mayores a $5 \mathrm{~m}$ y por el momento, rangos de marea menores a $5 \mathrm{~m}$ no son convenientes en virtud que su eficiencia es muy baja. La figura 1 muestra los sitios a nivel mundial que tienen mareas importantes, entre las cuales cabe destacar: Severn River (Reino Unido), Kimberleys (Australia), Cabo Tres Puntas (Argentina), Passamaquoddy (U.S.A.) y la bahia Fundy (Canada) (Charlier, 2002). Algunos de estos sitios ya cuentan con instalaciones como se muestra en la tabla 1, y otros sitios en estudio con buen potencial mareomotriz se muestran en la tabla 2 , donde se puede observar que México se encuentra entre estos sitios, con mareas del orden de $6 \mathrm{~m}$ en el alto Golfo de California. Corea y China han estudiado la posibilidad de construir

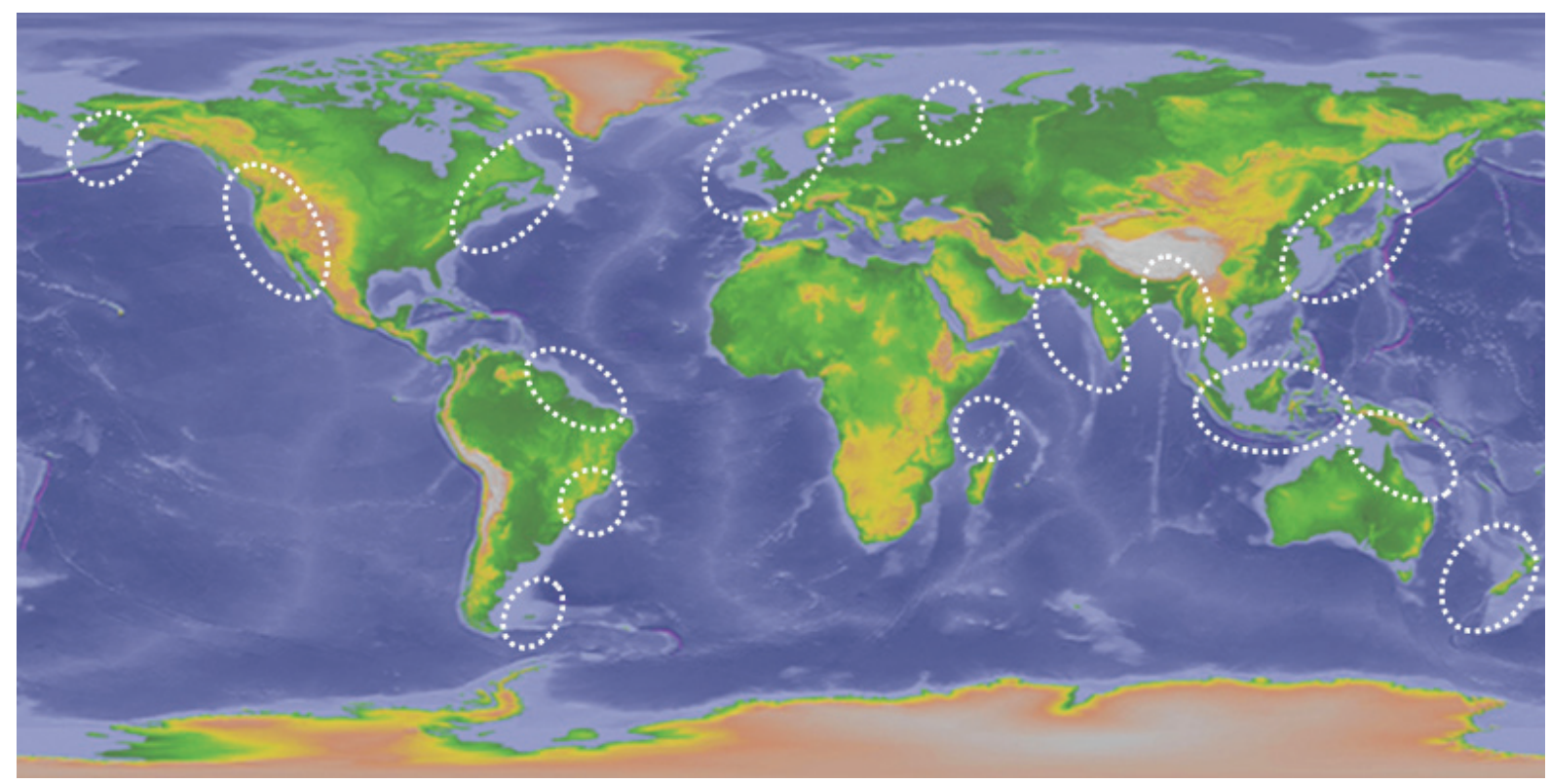

Figura 1. Sitios en el mundo con importantes rangos de marea 
DOI: http://dx.doi.org/10.22201/fi.25940732e.2010.11n2.019

Cuantificación de energía de una planta mareomotriz

algunas instalaciones y han estimado alrededor de 2,000 GWh por año de producción anual con plantas mareomotrices en Shiwa, Garolim y Incheom. Los estudios en China no son nuevos, en el reporte "Collection of Oceanographic Works" (2003) se presentó el potencial de energía mareomotriz para el periodo de 1950-1990.
Aunque hay varios lugares apropiados para aprovechar este tipo de energía y se cuenta con la ingeniería para hacerlo, aún existen muchos problemas por resolver como: impacto ambiental, factibilidad económica y despacho de energía de acuerdo a una curva de demanda (Charlier, 1988) y (Song WO, 1987).

Tabla 1. Instalaciones mareomotrices

\begin{tabular}{lcccccc}
\hline $\begin{array}{c}\text { Nombre de la } \\
\text { Maremotriz }\end{array}$ & $\begin{array}{c}\text { Fecha de puesta } \\
\text { en servicio }\end{array}$ & $\begin{array}{c}\text { Rango } \\
\text { Marea }(\mathrm{m})\end{array}$ & $\begin{array}{c}\text { Área del Embalse } \\
\left(\mathrm{km}^{2}\right)\end{array}$ & $\begin{array}{c}\text { Núm. de turbinas } \\
\text { instaladas }\end{array}$ & $\begin{array}{c}\text { Potencia instalada } \\
\text { MW }\end{array}$ & $\begin{array}{c}\text { Generación } \\
\text { GWh/año }\end{array}$ \\
\hline La Rance (Francia) & 1966 & 8 & 17 & 24 & 240 & 540 \\
$\begin{array}{l}\text { Anápolis Royal } \\
\text { (Canadá) }\end{array}$ & 1984 & 10.8 & 6 & 1 & 16 & 30 \\
Jiangxia (China) & 1980 & 7.1 & 2 & 1 & 3.2 & 11 \\
Kislaya Bay (Rusia) & 1968 & 2.4 & 2 & 1 & 0.4 & 3.5 \\
Severn (Reino Unido) & 2000 & 7 & 520 & en estudio & & \\
\hline
\end{tabular}

Tabla 2. Emplazamientos más destacados para el aprovechamiento de centrales maremotrices (continúa ...)

\begin{tabular}{|c|c|c|c|c|c|}
\hline \multirow[t]{2}{*}{ País } & \multirow[t]{2}{*}{ Emplazamiento } & \multirow{2}{*}{$\begin{array}{c}\text { Altura media } \\
\text { de marea } \\
\mathrm{m}\end{array}$} & \multirow{2}{*}{$\begin{array}{c}\text { Superficie } \\
\text { embalsada } \\
\text { km2 }\end{array}$} & \multirow{2}{*}{$\begin{array}{c}\text { Potencia } \\
\text { estimada } \\
\text { MW }\end{array}$} & \multirow{2}{*}{$\begin{array}{l}\text { Producción } \\
\text { aproximada } \\
\text { GWh/ANO }\end{array}$} \\
\hline & & & & & \\
\hline \multirow{3}{*}{ Argentina } & San José & 5.9 & 778 & 5040 & 9400 \\
\hline & Santa Cruz & 7.5 & 222 & 2420 & \\
\hline & Rio Gallegos & 7.5 & 177 & 1900 & \\
\hline \multirow{2}{*}{ Australia } & Bahía de Secure & 7 & 140 & 1480 & \\
\hline & Ensenada de Walcott & 7 & 260 & 2800 & \\
\hline \multirow{3}{*}{ Canadá } & Cobequid & 13.4 & 240 & 5338 & 14000 \\
\hline & Cumberland & 10.9 & 90 & 1400 & \\
\hline & Shepody & 10 & 115 & 1800 & \\
\hline \multirow{3}{*}{ Rusia } & Bahía de Mezen & 6.76 & 2640 & 15000 & 45000 \\
\hline & Penzhinsk, Mar de Okhost & 11.4 & 530 & 87400 & 190000 \\
\hline & Bahía de Tugur & 6.8 & 1080 & 7800 & 16200 \\
\hline \multirow{3}{*}{ USA } & Turnagain Arm & 7.5 & & 6500 & 16600 \\
\hline & Knit Arm & 7.5 & & 2900 & \\
\hline & Pasamaquoddy & 5.5 & & & \\
\hline \multirow{2}{*}{ India } & Golfo de Khambat & 6.8 & 1970 & 7000 & 15000 \\
\hline & Golfo de Kutch & 5 & 170 & 900 & \\
\hline \multirow{2}{*}{ UK } & Severn & 7 & 520 & 8640 & 17000 \\
\hline & Mersey & 6.5 & 61 & 700 & \\
\hline
\end{tabular}


DOI: http://dx.doi.org/10.22201/fi.25940732e.2010.11n2.019

López-González J., Hiriart-Le Bert G. y Silva-Casarín R.

Tabla 2. Emplazamientos más destacados para el aprovechamiento de centrales maremotrices (... continuación)

\begin{tabular}{cccccc}
\hline País & Emplazamiento & $\begin{array}{c}\text { Altura media } \\
\text { de marea } \\
\mathrm{m}\end{array}$ & $\begin{array}{c}\text { Superficie } \\
\text { embalsada } \\
\mathrm{km} 2\end{array}$ & $\begin{array}{c}\text { Potencia } \\
\text { estimada } \\
\mathrm{MW}\end{array}$ & $\begin{array}{c}\text { Producción } \\
\text { aproximada } \\
\text { GWh/AÑO }\end{array}$ \\
\hline \multirow{3}{*}{ UK } & Duddon & 5.6 & 20 & 100 & \\
& Wyre & 6 & 5.8 & 64 & \\
\hline \multirow{2}{*}{ Corea } & Conwy & 5.2 & 5.5 & 400 \\
\multirow{2}{*}{ México } & Garolim & 4.7 & 100 & \\
\hline
\end{tabular}

Extracto de: Fernández-Díez P. Energía Mareomotriz. Departamento de Ingeniería

Eléctrica y Energética. Universidad de Catabria. Disponible en:

http://www.termica.webhob.info/

\section{Estimación de la potencia disponible}

La energía potencial puede ser estimada con un modelo paramétrico, conociendo las características de la marea como: altura o las principales componentes armónicas, además de algunos coeficientes que en muchos casos contienen mucha incertidumbre (Charlier, 2003).

En este trabajo se presenta una forma alternativa para realizar una evaluación preliminar, la cual consiste en seleccionar la altura de diseño, el área del embalse y la potencia instalada. Por medio de una grafica se puede estimar la cantidad de energía que se producirá con esta combinación.

La energía potencial de la marea es directamente proporcional al rango de marea. En el caso particular de una mareomotriz, es la diferencia entre la elevación del nivel del mar y el nivel del embalse. Con la ecuación (1) es posible calcular la potencia de una instalación.

$$
P=\gamma Q H
$$

donde:

$H$ Es la diferencia de niveles entre el embalse y el mar, expresada en metros.

Q Gasto que pasa a través de las turbinas en $\mathrm{m}^{3} / \mathrm{s}$.

$\gamma$ El peso especifico igual a la densidad por la gravedad por $\mathrm{m}^{3}$.

Q Puede ser calculado como:

$$
Q=A V
$$

donde:

A Es el área transversal de las turbinas (m2).

$V$ Velocidad media en el área transversal de las turbinas $(\mathrm{m} / \mathrm{s})$.

$V$ Puede ser calculada como:

$$
V=C d \sqrt{2 g H}
$$

\section{$C d$ Coeficiente de pérdidas}

Cuando la energía cambia o entra a un sistema, una parte de esta energía se pierde, debido a muchos factores, como pérdidas de fricción a lo largo de la trayectoria y cambios bruscos de sección y dirección entre otros, lo cual se puede tomar en cuenta con un factor de eficiencia a través de la relación entre la energía transformada y la energía disponible. Entonces, la ecuación de potencia quedaría de la siguiente manera:

$$
P=\eta \rho A \sqrt{2 g} H^{3 / 2}
$$

\section{$\eta$ Factor de eficiencia incluye}

Teniendo los datos de marea se puede conocer el nivel del mar en cualquier instante; sin embargo, el nivel en el embalse depende de cuánta agua está entrando o saliendo, lo cual depende del volumen de almacenamiento y gasto de descarga, que a su vez, depende del número de turbinas y su capacidad, así como de la altura entre un lado y otro del embalse $(\Delta H)$. 
Se hizo un modelo numérico para simular el funcionamiento de una mareomotriz con datos discretos de marea a cada 15 min del puerto de San Felipe, en el alto Golfo de California (Mar de Cortés). Los datos de marea se muestran en figura 2 , donde se aprecia un rango máximo de marea de $6 \mathrm{~m}$ aproximadamente.

Suponiendo una determinada área del embalse y seleccionando la potencia instalada fue posible calcular la energía de generación anual para esta combinación, posteriormente se modificó la potencia instalada y nuevamente se calculó la generación, de esta manera se construyó una curva de potencia instalada contra generación por kilometro cuadrado.

Repitiendo la operación antes mencionada, se construyeron las curvas de potencia instalada contra generación para las diferentes formas de operación: Flujo, Flujo-reflujo y doble embalse.

Estas curvas se muestran en la figura 3. Con estas curvas es posible seleccionar cualquier valor de potencia instalada por kilometro cuadrado y conocer la generación anual para el Alto Golfo de California.
De las curvas de la figura 3 es posible estimar la cantidad de energía de generación para cualquier combinación de potencia instalada y área del embalse para las diferentes formas de generación.

Hay que aclarar que para calcular la cantidad de energía se utiliza el área de la mareomotriz, por lo que en el caso del doble embalse el área a utilizar es el de los dos embalses.

El ejercicio de la tabla 3 se muestra la forma de cómo utilizar estas curvas con la misma área de la mareomotriz y misma potencia instalada.

Con la misma área y misma potencia instalada, el esquema de un solo embalse podría ser una mejor opción, ya que se obtiene más energía.

Sin embargo, existen intervalos sin generación, debido a que no hay desnivel entre el embalse y la superficie del mar.

Por el contrario, con el esquema de doble embalse es posible despachar la energía de una manera más uniforme (figura 4), por lo cual, el esquema de doble embalse se vislumbra como el más recomendable.

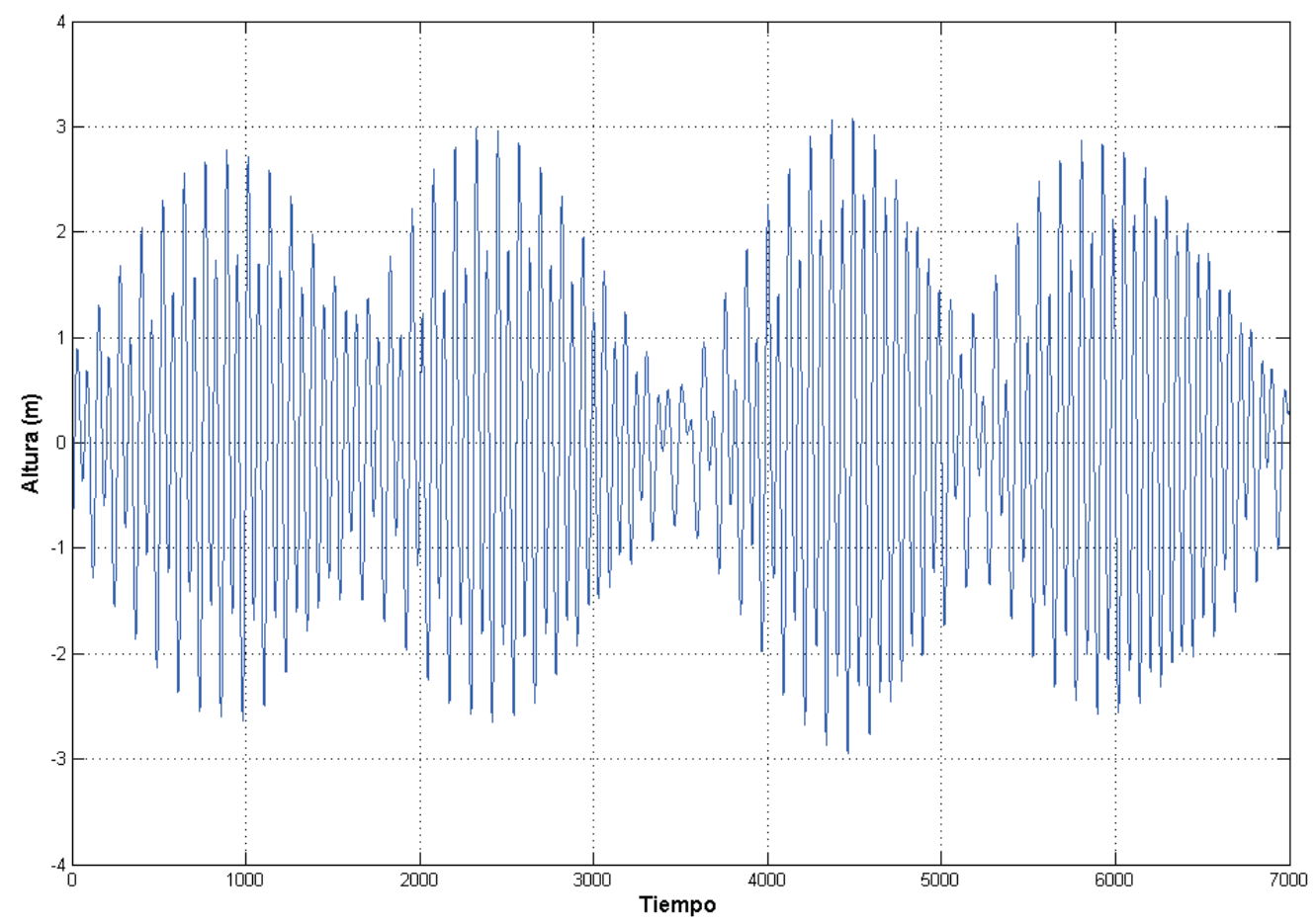

Figura 2. Marea en San Felipe 


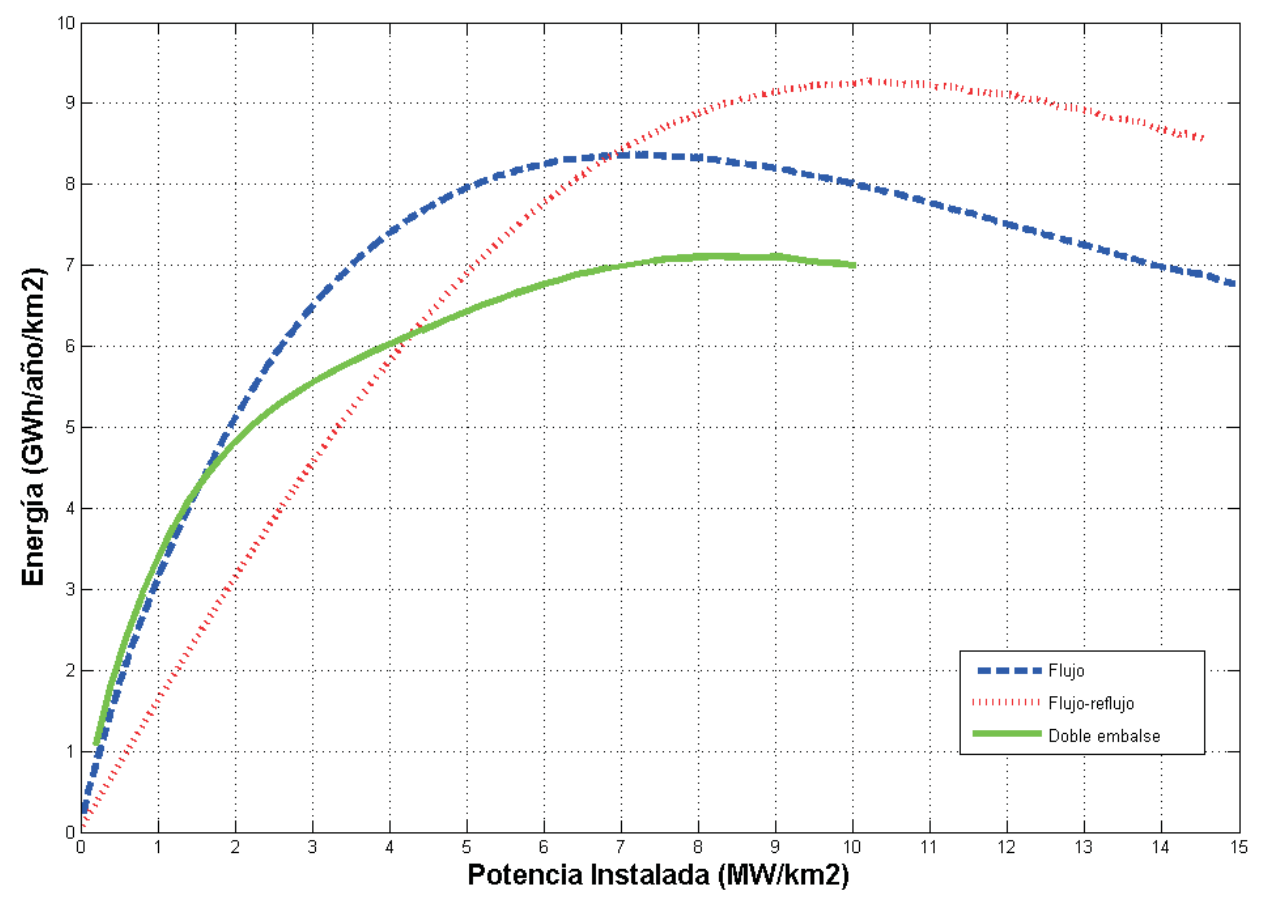

Figura 3. Generación en San Felipe

Tabla 3. Utilización de curvas con la misma área de la mareo motriz y potencia instalada

\begin{tabular}{|c|c|c|c|c|c|}
\hline & $\begin{array}{l}\text { Área } \\
\left(\mathrm{km}^{2}\right)\end{array}$ & $\begin{array}{c}\text { Potencia instalada } \\
(\mathrm{MW})\end{array}$ & $\begin{array}{c}\text { Índice del eje } \\
\text { de abscisas }\end{array}$ & $\begin{array}{c}\text { Índice en el } \\
\text { eje de las ordenadas }\end{array}$ & $\begin{array}{l}\text { Generación } \\
\text { GWh/año }\end{array}$ \\
\hline $\begin{array}{l}\text { Un embalse } \\
\text { modo de flujo }\end{array}$ & 50 & 250 & $250 / 50=5$ & 8 & $8 \times 50=400$ \\
\hline $\begin{array}{c}\text { Doble embalse } \\
\text { Área } 50 \mathrm{~km}^{2}\end{array}$ & $\begin{array}{c}50 \\
\text { (un solo embalse 25) }\end{array}$ & 250 & $250 / 50=5$ & 6.5 & $6.5 \times 50=345$ \\
\hline
\end{tabular}
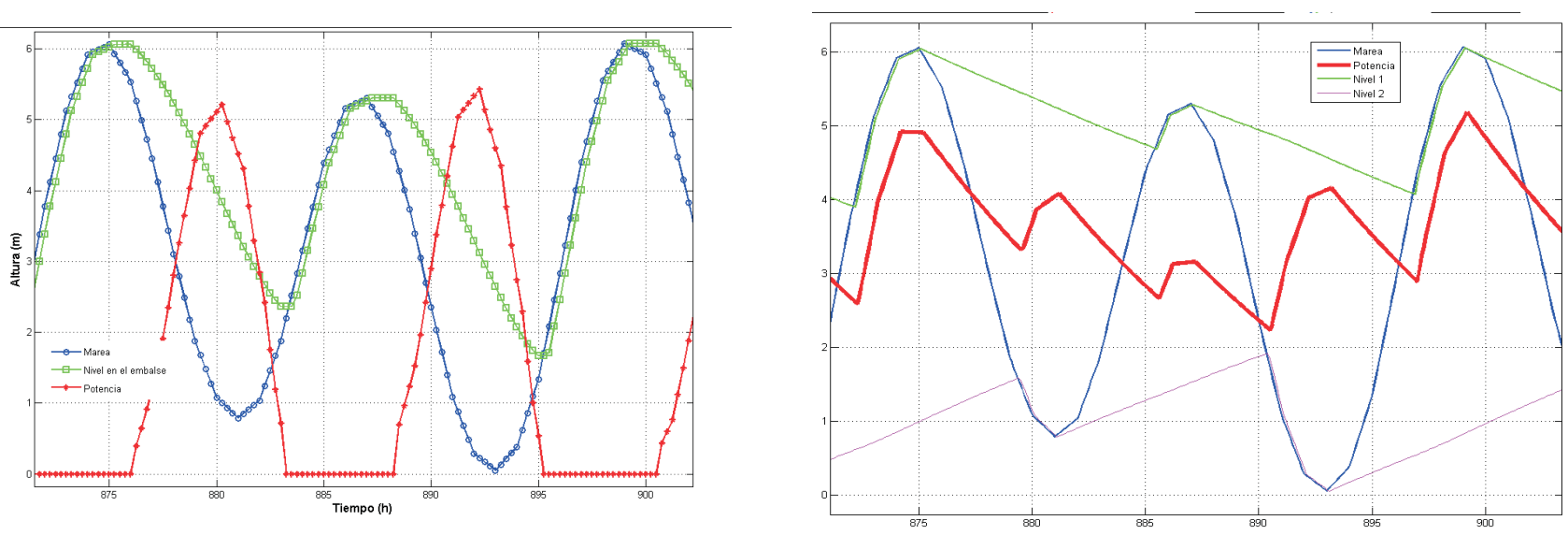

Figura 4. Generación con un embalse en modo de reflujo y generación con doble embalse (azul marea, verde nivel en el embalse, rojo potencia) 
DOI: http://dx.doi.org/10.22201/fi.25940732e.2010.11n2.019

\section{Generalización}

Con la figura 3 es posible conocer la generación en San Felipe, por la potencia instalada y el área del embalse. En forma general, para conocer la generación en diferentes partes del mundo, se utilizó un espectro de energía en el dominio del periodo para 3 sitios: San Felipe en México, La Rance Francia y Puerto Mont en Chile. Los tres sitios tienen marea semidiurna y el principal componente armónico es el Lunar $\mathrm{M}_{2}$, como se puede observar en la figura 5. Los diferentes picos que aparecen en esta gráfica se deben a que el intervalo de las muestras (Nyquist) no es un múltiplo de las frecuencias (Emery et al., 2004). Sin embargo, en la tabla 4 se puede observar que el único componente cercano a estos picos es la componente $\mathrm{M}_{2}$ con periodo de $12.42 \mathrm{~h}$.

Se puede inferir que es posible construir una sola curva para conocer la generación, ya que la única diferencia entre los tres sitios es la amplitud de marea. Una posibilidad es tomar las tres principales componentes armónicas y otra es utilizar un promedio de amplitud de marea (raíz media cuadrática), lo cual es sencillo de hacer.

Al igual que en San Felipe, con el modelo numérico se simuló la generación para Puerto Mont y La Rance y se construyeron curvas de potencia instalada contra generación para los diferentes modos de operación; estas graficas se muestran en las figuras 6 y 7 .
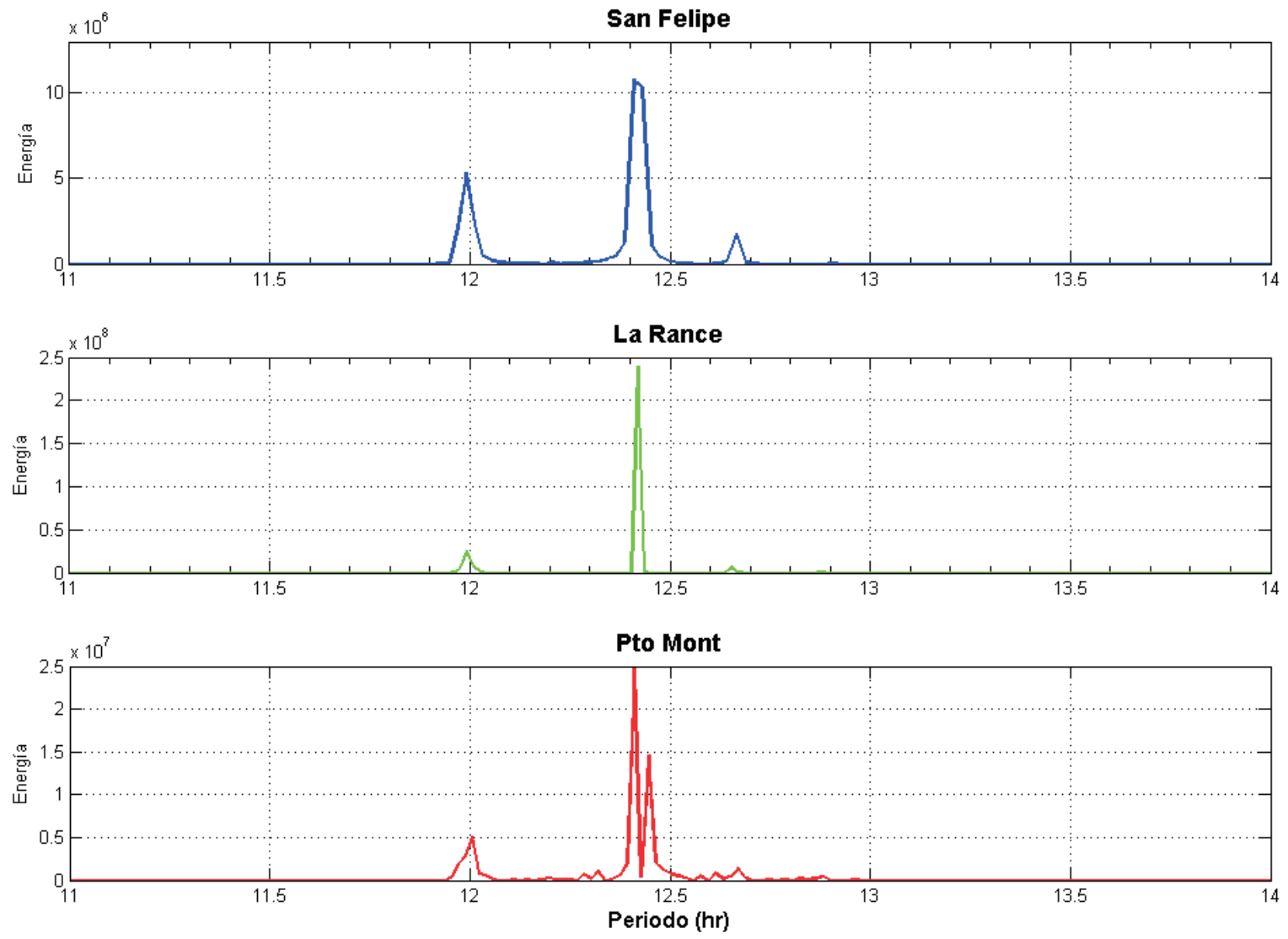

Figura 5. Espectro de energía 
DOI: http://dx.doi.org/10.22201/fi.25940732e.2010.11n2.019

López-González J., Hiriart-Le Bert G. y Silva-Casarín R.

Tabla 4. Principales componentes armónicas de la marea

\begin{tabular}{clccc}
\hline Núm. & $\begin{array}{c}\text { Nombre de la } \\
\text { componente armónica }\end{array}$ & Símbolo & Periodo $(\mathrm{hr})$ & $\% \mathrm{M}_{2}$ \\
\hline 1 & Principal lunar & $\mathrm{M}_{2}$ & 12.42 & 100.0 \\
2 & Lunisolar diurna & $\mathrm{K}_{1}$ & 23.93 & 58.4 \\
3 & Principal solar & $\mathrm{S}_{2}$ & 12.00 & 46.6 \\
4 & Principal lunar diurna & $\mathrm{O}_{1}$ & 25.82 & 41.5 \\
5 & Principal solar diurna & $\mathrm{P}_{1}$ & 24.07 & 19.4 \\
6 & Lunar elíptica Mayor & $\mathrm{N}_{2}$ & 12.66 & 19.2 \\
7 & Lunar quincenal & $\mathrm{M}_{\mathrm{f}}$ & 327.67 & 17.2 \\
8 & Lunisolar semidiurna & $\mathrm{K}_{2}$ & 11.97 & 12.7 \\
9 & Lunar mensual & $\mathrm{M}_{\mathrm{m}}$ & 661.30 & 9.1 \\
10 & Solar semi anual & $\mathrm{S}_{\mathrm{sa}}$ & 2191.43 & 8.0 \\
\hline
\end{tabular}

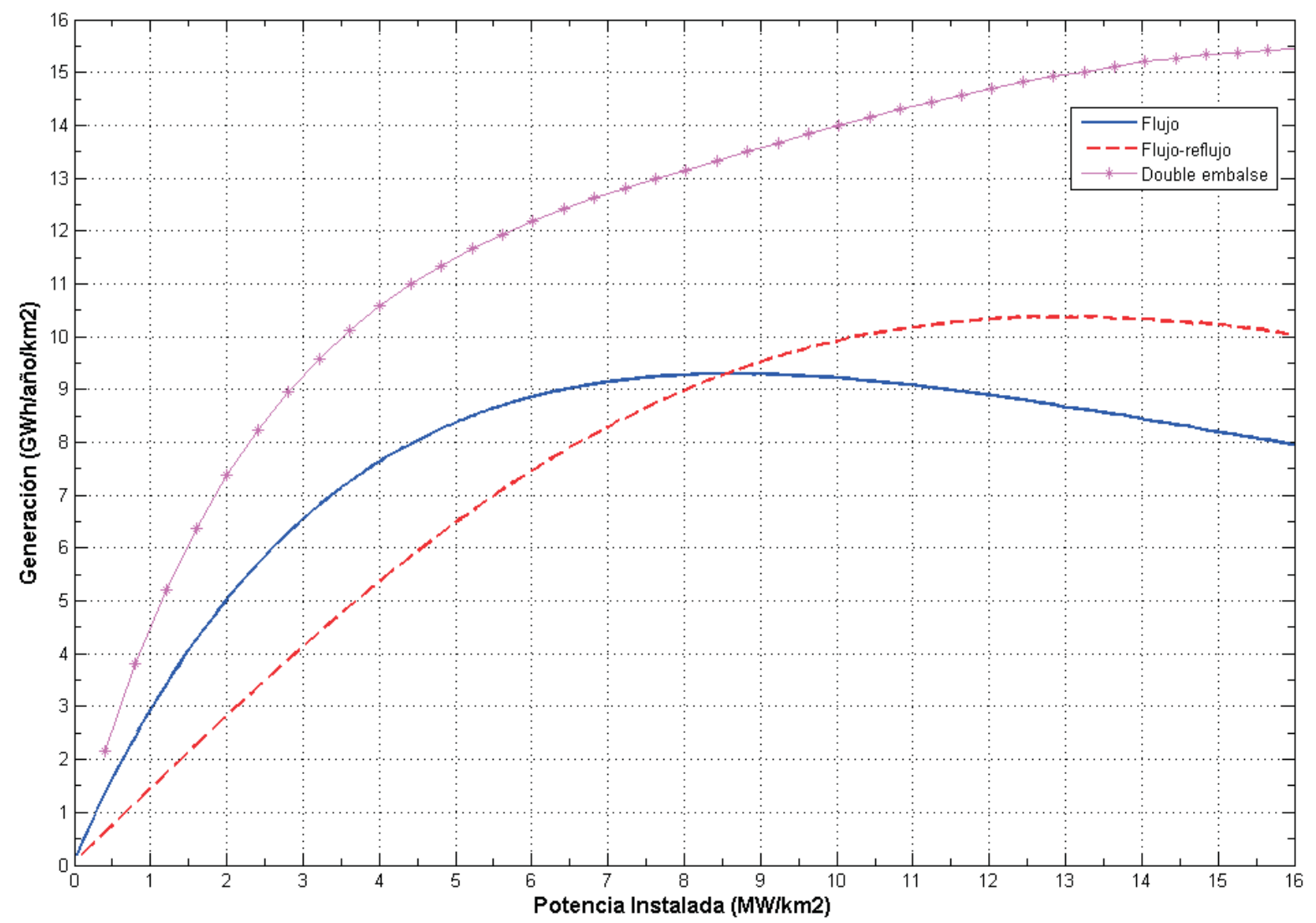

Figura 6. Generación en Puerto Mont (Chile) 


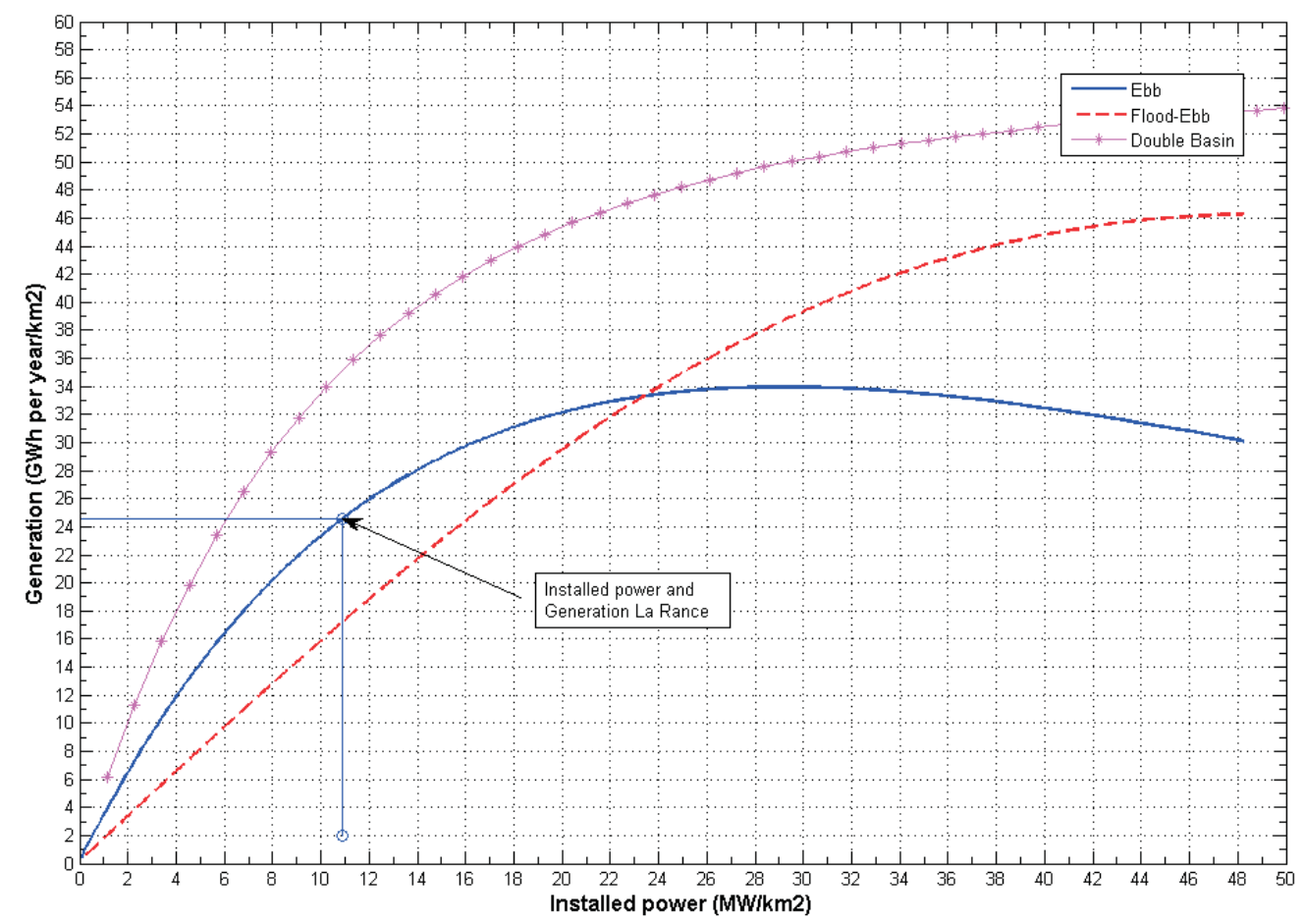

Figura 7. Generación en La Rance

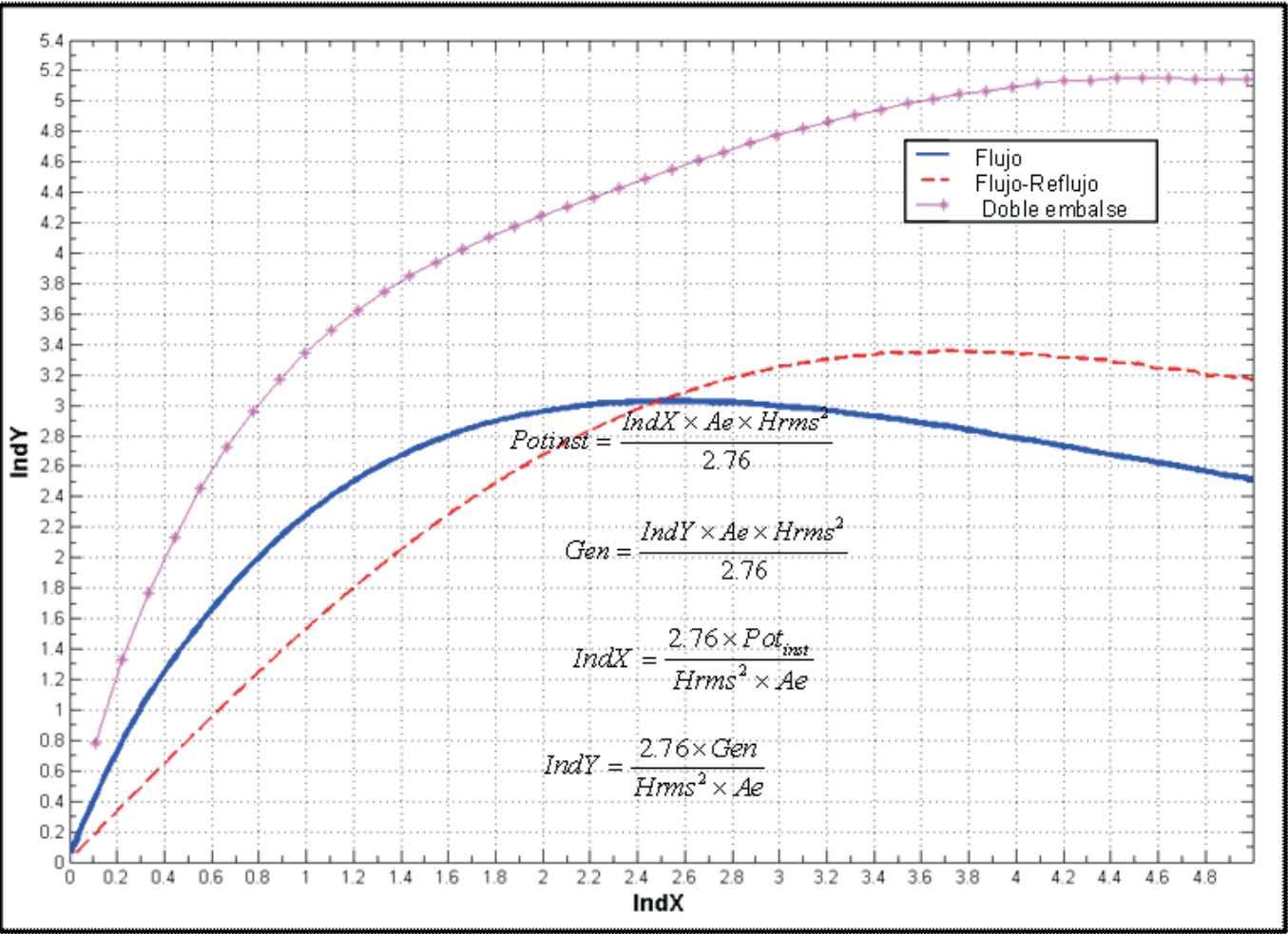

Figura 8. Curvas normalizadas para conocer la generación en una planta mareomotriz 
DOI: http://dx.doi.org/10.22201/fi.25940732e.2010.11n2.019

López-González J., Hiriart-Le Bert G. y Silva-Casarín R.

Fácilmente, se puede observar que las curvas presentadas en las figuras 3,6 y 7 tienen la misma forma, solo que a otra escala, por lo tanto, para extender los resultados se buscó la forma de hacer unitaria una de las graficas y escalarla de acuerdo al sitio de interés. Se tomaron las curvas de San Felipe y se hicieron unitarias dividiendo entre la altura media cuadrática de la marea. De esta forma si se quieren construir las otras dos curvas sólo es necesario multiplicar por la altura cuadrática media del sitio de interés. Estas curvas se muestran en la figura 8 y a través de ellas es posible obtener los mismos resultados para los tres sitios indistintamente. Por ejemplo, para la mareomotriz de La Rance que tiene un área de 22 $\mathrm{km}^{2}$ y una producción de $240 \mathrm{MWh} /$ año y el modo de generación es reflujo, se puede obtener de esta gráfica que la generación es de $550 \mathrm{GWh} /$ año, lo cual es muy similar a lo reportado de $500 \mathrm{GWh} / a n ̃ o$ (Romanoski, $1950=y$ (Charlier et al., 1993).

\section{Consideraciones}

A continuación se presentan los datos que se consideraron para las simulaciones.

\begin{tabular}{cccc}
\hline Sitio & Hrms & Marea máx. & Carga \\
\hline San Felipe & 2.76 & 6 & 3.60 \\
Puerto Mont & 3.00 & 7 & 4.20 \\
La Rance & 5.60 & 13 & 8.00 \\
\hline
\end{tabular}

Para verificar la robustez del método propuesto se realizó una comparación entre las curvas obtenidas con el modelo numérico y la curva normalizada de la figura 8 , los resultados se presentan en la tabla 5 . El error obtenido para el caso de generación en modo de flujo y flujo-reflujo, es menor al 10\%, por lo cual, los resultados con esta gráfica se pueden considerar adecuados y útiles para una primera evaluación del recurso.

Para obtener la energía de generación de la mareomotriz en el caso del doble embalse, en la curva normalizada el área considerada tendrá que ser la que corresponde a un solo embalse.

\section{Conclusiones}

El potencial de energía disponible en el océano, asociada a las mareas autonómicas es muy importante. Para valorar la capacidad es muy importante tener en cuenta la amplitud de la marea, el número de turbinas instaladas y el área del embalse, ya que estos son factores que determinan la cantidad de energía disponible. Una combinación adecuada de estos tres factores dará como resultado los mayores beneficios.

En este trabajo se presenta una metodología confiable y de fácil aplicación para conocer la cantidad de energía aprovechable, dependiendo del área del embalse, la potencia instalada y la amplitud de la marea, la cual puede ser útil para evaluar proyectos de factibilidad de esta naturaleza.

Tabla 5. Comparación entre el modelo numérico y las curvas normalizadas, considerando diferentes esquemas de generación (continúa...)

\begin{tabular}{|c|c|c|c|c|c|c|c|c|c|c|c|c|c|c|c|}
\hline \multirow{3}{*}{ Sitio } & \multirow{3}{*}{ Curva } & \multirow{3}{*}{$\begin{array}{c}\text { Potencia } \\
\text { MW }\end{array}$} & \multirow{3}{*}{$\begin{array}{l}\text { Área } \\
\mathrm{Km}^{2}\end{array}$} & \multicolumn{4}{|c|}{ Modo de flujo } & \multicolumn{4}{|c|}{ Modo de flujo Reflujo } & \multicolumn{4}{|c|}{ Doble embalse } \\
\hline & & & & ind $X$ & ind $Y$ & Energía & error & ind $X$ & ind $Y$ & Energía & error & ind $\mathrm{X}$ & ind $Y$ & Energía & error \\
\hline & & & & & & $\mathrm{GWh} / \mathrm{y}$ & & & & GWh/y & & & & GWh/y & \\
\hline \multirow[t]{6}{*}{ San Felipe } & Modelo & 200 & 50 & 4 & 7.5 & 375 & & 4 & 5.9 & 295 & & 2 & 7.9 & 198 & \\
\hline & Modelo & 150 & 60 & 2.5 & 6 & 360 & & 2.5 & 4 & 240 & & 1.25 & 5.8 & 174 & \\
\hline & Modelo & 540 & 60 & 9 & 8.1 & 486 & & 9 & 9.1 & 546 & & 4.5 & 11.2 & 336 & \\
\hline & Normalizada & 200 & 50 & 1.45 & 2.7 & 373 & $-0.6 \%$ & 1.45 & 2.1 & 290 & $-1.8 \%$ & 0.72 & 2.8 & 193 & $-2.2 \%$ \\
\hline & Normalizada & 150 & 60 & 0.91 & 2.15 & 356 & $-3.4 \%$ & 0.91 & 1.4 & 232 & $-3.4 \%$ & 0.45 & 2.2 & 182 & $4.7 \%$ \\
\hline & Normalizada & 540 & 60 & 3.26 & 2.95 & 489 & $0.1 \%$ & 3.26 & 3.3 & 546 & $0.1 \%$ & 1.63 & 4 & 331 & $-1.4 \%$ \\
\hline \multirow[t]{3}{*}{ Pto Mont } & Modelo & 200 & 50 & 4 & 7.6 & 380 & & 4 & 5.4 & 270 & & 2 & 7.5 & 188 & \\
\hline & Modelo & 150 & 60 & 2.5 & 5.7 & 342 & & 2.5 & 3.5 & 210 & & 1.25 & 5 & 150 & \\
\hline & Modelo & 540 & 60 & 9 & 9.3 & 558 & & 9 & 9.6 & 576 & & 4.5 & 11.1 & 333 & \\
\hline
\end{tabular}


DOI: http://dx.doi.org/10.22201/fi.25940732e.2010.11n2.019

Cuantificación de energía de una planta mareomotriz

Tabla 5. Comparación entre el modelo numérico y las curvas normalizadas, considerando diferentes esquemas de generación (... continuación)

\begin{tabular}{|c|c|c|c|c|c|c|c|c|c|c|c|c|c|c|c|}
\hline \multirow{3}{*}{ Sitio } & \multirow{3}{*}{ Curva } & \multirow{3}{*}{$\begin{array}{c}\text { Potencia } \\
\text { MW }\end{array}$} & \multirow{3}{*}{$\begin{array}{l}\text { Área } \\
\mathrm{Km}^{2}\end{array}$} & \multicolumn{4}{|c|}{ Modo de flujo } & \multicolumn{4}{|c|}{ Modo de flujo Reflujo } & \multicolumn{4}{|c|}{ Doble embalse } \\
\hline & & & & ind $X$ & indY & Energía & error & indX & ind $Y$ & Energía & error & ind $\mathrm{X}$ & ind $Y$ & Energía & error \\
\hline & & & & & & $\mathrm{GWh} / \mathrm{y}$ & & & & GWh/y & & & & GWh/y & \\
\hline \multirow[t]{3}{*}{ Pto Mont } & Normalizda & 200 & 50 & 1.23 & 2.5 & 408 & $7.3 \%$ & 1.23 & 1.81 & 295 & $9.3 \%$ & 0.61 & 2.6 & 212 & $13.0 \%$ \\
\hline & Normalizada & 150 & 60 & 0.77 & 1.9 & 372 & $8.7 \%$ & 0.77 & 1.2 & 235 & $11.8 \%$ & 0.38 & 1.8 & 162 & $8.0 \%$ \\
\hline & Normalizada & 540 & 60 & 2.76 & 3.1 & 607 & $8.7 \%$ & 2.76 & 3.19 & 624 & $8.4 \%$ & 1.38 & 3.8 & 342 & $2.7 \%$ \\
\hline \multirow[t]{6}{*}{ La Rance } & Modelo & 200 & 50 & 4 & 12.5 & 625 & & 4 & 7 & 350 & & 2 & 10 & 250 & \\
\hline & Modelo & 150 & 60 & 2.5 & 8 & 480 & & 2.5 & 4 & 240 & & 1.25 & 7 & 210 & \\
\hline & Modelo & 540 & 60 & 9 & 22 & 1320 & & 9 & 15 & 900 & & 4.5 & 20 & 600 & \\
\hline & Normalizada & 200 & 50 & 0.35 & 1.2 & 682 & $9.1 \%$ & 0.35 & 0.58 & 330 & $-5.9 \%$ & 0.18 & 1.1 & 280 & $12.0 \%$ \\
\hline & Normalizada & 150 & 60 & 0.22 & 0.8 & 545 & $13.6 \%$ & 0.22 & 0.35 & 239 & $-0.6 \%$ & 0.11 & 0.8 & 225 & $7.1 \%$ \\
\hline & Normalizada & 540 & 60 & 0.79 & 2 & 1363 & $3.3 \%$ & 0.79 & 1.25 & 852 & $-5.3 \%$ & 0.40 & 2 & 652 & $8.7 \%$ \\
\hline
\end{tabular}

En México el potencial de energía mareomotriz en el alto Golfo de California puede llegar a ser muy importante si consideramos una barrera colocada en la cota -10 , la cual tendría dos funciones servir de cortina para el embalse y como vía de comunicación, dando como resultado un área de embalse de $2590 \mathrm{~km}^{2}$, potencia máxima de acuerdo a la Gráfica 3 en modo de flujo-reflujo de 26 GW y una producción de $23000 \mathrm{GWh}$ /año que representa más o menos la producción de todas las centrales hidroeléctricas del país, obviamente la colocación de esta cantidad de turbinas es exagerada; sin embargo, la producción de energía eléctrica puede ser importante, aún colocando solo una fracción de ésta.

En cuanto a cantidad y despacho de energía se refiere, el esquema de doble embalse se aprecia como viable, en este caso, sería importante evaluar el costo económico y social, así como el impacto ecológico. Cabe señalar que el modelo numérico toma en cuenta los principales factores de generación pero en condiciones ideales. Para una evaluación real es necesario ajustar los parámetros y coeficientes de acuerdo a las condiciones de cada sitio en particular.

\section{Agradecimientos}

Al proyecto IMPULSA4 "Desalación de agua de mar con energías renovables" y al Coordinador de proyecto Sergio Alcocer Martínez de Castro. Al Consejo Nacional de Ciencia y Tecnología (CONACYT).

\section{Referencias}

Ballenilla F. El final del petróleo barato. El ecologista No. 40. Alicante España. 2004.

Charlier R.H. Justus J.R. Ocean Energies Environmental, Economic and Technological Aspects of Alternative Power Sources. Elsevier Oceanography Series 56. 1993.

Charlier R.H. Tidal Energy. Van Nostrand-Reinhold. New York. 1982.

Charlier R.H. A "Sleeper" Awakes: Tidal Current Power. Journal of Renew Sustainable Energy, 2003.

Charlier R.H. Resurgimento or Aggiornamento? Tidal Power at 30 Years. Journal of Renew Sustainable Energy. 1(4): 271-89. 2002.

Charlier R.H. Ocean Alternative Energy. The View From China_'Small is Beautiful'. Journal of Renew Sustainable Energy.

Charlier R.H., Me'nanteau L. The saga of tide mills. Journal of Renewable Sustainable Energy, 1(3):1-44. 1998.

Dincer I. Renewable Energy and Sustainable Development: a Crucial Review, Renewable and Sustainable Energy Reviews. Elsevier. 2000.

Emery and Thomson. Data Analysis Methods in Physical Oceanography. Elsevier Oceanography Series. 2004.

Fells I. The problem. In: Dunderdale J, editor. Energy and the Enviroment. UK: Royal Society of Chemistry. 1990.

Fernández-Díez P. Energía mareomotriz. Departamento de Ingeniería Eléctrica y Energética. Universidad de Catabria 
DOI: http://dx.doi.org/10.22201/fi.25940732e.2010.11n2.019

López-González J., Hiriart-Le Bert G. y Silva-Casarín R.

[en línea]. Disponible en: http://www.termica.webhob.info/

Isaacs J.D., Seymour R.J. (1973). The Ocean as a Power Resource. International Journal of Environmental Studies, 4(3). 2001.

Kwang-Soo L. Ocean Energy Activities in Korea Ocean Energy Activities in Korea (Tidal Barrage \& Tidal Current).
International Seminar on Water and Energy, UNAM, Mexico City, pp. 22-23, March 2007.

Romanoski V. Les Energies de la Mer (Collection Que sais-je?). University Press of France, Paris. 1950.

Song WO. Reassessment of the Garolim tidal power project. Ocean Research (Korea), 9(1/2):29-35. 1987.

\section{Semblanza de los autores}

José López-González. Estudiante de doctorado, obtuvo el grado de maestro en ingeniería en la Universidad Nacional Autónoma de México (UNAM), con especialidad en hidráulica marítima. Con 15 años de experiencia en consultoría de procesos e ingeniería costera es profesor de la Facultad de Ingeniería en la UNAM. Actualmente estudia las fuentes de energía renovable del océano y desarrolla un dispositivo de conversión de energía marina dentro del proyecto IMPULSA "Desalación de agua de mar con energías renovables".

Gerardo Hiriart-Le Bert. Doctor en ingeniería mecánica por la Naval Postgraduate School de Monterey, California, Estados Unidos de América. Es investigador en el Instituto de Ingeniería de la Universidad Nacional Autónoma de México (UNAM) y jefe del proyecto IMPULSA "Desalación de agua de mar con energías renovables". Es miembro director del Consejo del Circumpacifico para la energía y recursos minerales en donde preside la Comisión de Energías Renovables; laboró en el área de geotermia y energías renovables de la Comisión Federal de Electricidad (México). Ha sido distinguido con el premio "Los Pioneros" por el Consejo de Recursos Geotérmicos de Estados Unidos de América y con el Premio Nacional de Energías Renovables por la Comisión Nacional para el Ahorro de Energía (CONAE) de México.

Rodolfo Silva-Casarín. En 1995 obtuvo el grado de doctor en ingeniería de caminos, canales y puertos en la Universidad de Cantabria, España. Actualmente es investigador de tiempo completo en el Instituto de Ingeniería y profesor titular en la Facultad de Ingeniería (División de Estudios de Posgrado de la Facultad de Ingeniería UNAM). Ha impartido 26 cursos y dirigido varias tesis de licenciatura, maestría y doctorado. Ha realizado una cantidad importante de publicaciones científicas tanto nacionales como extranjeras. Dentro de las distinciones recibidas se encuentra el Premio Nacional "Diario de México", al mejor estudiante de México en 1990. Es miembro de varias asociaciones académicas y científicas y ha participado como árbitro y colaborador científico en diferentes revistas, consejos, comités y evaluador de estímulos de carrera entre otras, así como, árbitro evaluador del CONACYT. 